\title{
Research on the Protection and Development of Important Agricultural Cultural Heritage in China
}

\author{
Long Hua ${ }^{1}$, Yang Bo ${ }^{2}$, Zhong Ting ${ }^{3}$, Chen Meinan ${ }^{4}$, Li Ruoyu ${ }^{4}$ \\ ${ }^{1}$ College of Agronomy and Biotechnology, Yunnan Agricultural University, Yunnan Kunming \\ 650201, China \\ ${ }^{2}$ Department of foreign languages, Kunming Medical University, Yunnan Kunming, 650500, China \\ ${ }^{3}$ Kunming tourism vocational school, Kunming tourism vocational school \\ ${ }^{4}$ College of Longrun Pu-erh Tea, Yunnan Agricultural University, Kunming 650201, China
}

Keywords: China, important agricultural cultural heritage, protection, development

Abstract: In 2002, the United Nations Food and Agriculture Organization and the relevant international organization-level countries launched the Global Important Agricultural Cultural Heritage Protection Initiative. In 2005, China's Zhejiang Qingtian rice-fish symbiosis system was listed as China's first and the world's first batch of globally important agricultural cultural heritage protection pilots. This paper analyzes the concepts and characteristics of important agricultural cultural heritage, studies the important agricultural cultural heritage protection policies and mechanisms, the important agricultural cultural heritage dynamic protection mechanism, and explores the development path of important agricultural cultural heritage.

\section{Introduction}

The agricultural production system with agricultural biodiversity, traditional knowledge, traditional agricultural production techniques and cultural landscapes that our people have inherited in the long-term development process is an important agricultural cultural heritage. After the United Nations Food and Agriculture Organization (FAO) and relevant international organization-level countries launched the Global Important Agricultural Cultural Heritage Protection Initiative, China has actively influenced and participated in the protection of important global agricultural cultural heritage. As early as 2005, China's Zhejiang Qingtian rice-fish symbiosis system was listed as China's first and the world's first batch of important agricultural cultural heritage protection pilots. At this stage, 15 projects such as Yunnan Honghe Hani Rice Terraced Field System, Yunnan Pu'er Ancient Tea Garden and Tea Culture System have been included in the pilot of global important agricultural cultural heritage protection. Afterwards, the researchers in-depth study of the concept and connotation of agricultural cultural heritage, protection system and management system, protection and development path, and achieved certain results, which is of great significance to promote the protection of agricultural cultural heritage in China. 


\section{Concepts and Characteristics of Important Agricultural Cultural Heritage}

In a broad sense, agricultural cultural heritage is an agricultural related factor other than modern agriculture; from a narrow perspective, agricultural cultural heritage is an agricultural element that has not been used for agricultural production activities. Agricultural cultural heritage mainly includes material agricultural cultural heritage and non-material agricultural cultural heritage.

After proposing the Global Important Agricultural Cultural Heritage Protection Initiative, the agricultural cultural heritage has received the attention of all countries. Agricultural cultural heritage can also be called agricultural heritage, referring to material and cultural heritage and intangible cultural heritage related to agriculture; agricultural cultural heritage can also refer to sustainable agricultural production systems and landscapes, which emphasize agricultural biodiversity and systematic. Agricultural cultural heritage is different from the characteristics of world cultural heritage. Agricultural cultural heritage has the characteristics of natural heritage, cultural heritage, cultural landscape heritage, etc. Simply speaking, agricultural cultural heritage has the characteristics of activity, dynamics, adaptability, compounding and sustainability. From the perspective of folklore, China's agricultural cultural heritage has the characteristics of regional diversity and ethnic diversity.

\section{Important Agricultural Cultural Heritage Protection Policies and Mechanisms}

\subsection{Formulate an Important Agricultural Cultural Heritage Protection System}

In order to ensure the smooth protection of important agricultural cultural heritage, some scholars believe that in protecting important agricultural cultural heritage, it is not only necessary to follow international conventions, but also to protect the environmental resources and the protection of intangible cultural heritage in the region, establish a corresponding protection system. In addition, it is necessary to protect important agricultural cultural heritage by establishing local regulations. Some scholars believe that in order to improve the protection effect of important agricultural cultural heritage, the Regulations on the Protection of Agricultural Cultural Heritage can be formulated, and the definitions of important agricultural cultural heritage, the attribution of ownership, and the management mechanism are clearly defined in the Regulations. It can be formulated in accordance with other heritage laws and regulations. These have laid the foundation for the formulation and promulgation of the "Management Measures for Important Agricultural Cultural Heritage".

China has also thoroughly studied the identification criteria and identification principles of important agricultural cultural heritage, and pointed out the methods of census of important agricultural cultural heritage. In order to stimulate the enthusiasm of the people to protect important agricultural cultural heritage, China has been studying the ecological compensation mechanism. The study found that the agro-ecosystem service function has external characteristics, but there are widespread underestimation of the comprehensive benefits of eco-farming farming methods. Therefore, China is constantly improving the ecological compensation and reward system, and also assessing the ecological value of important agricultural cultural heritage sites, developing ecological compensation standards, China has proposed ecological compensation standards development plan and compensation path.

\subsection{Important Agricultural Cultural Heritage Management Mechanism}

Important agricultural cultural heritage involves a wide range of aspects, so the protection of agricultural cultural heritage will affect regional economic development. At this stage, the regional 
management methods and mechanisms adopted by China cannot effectively enhance the protection effect of important agricultural cultural heritage. Therefore, China needs to constantly improve its management mechanism and clearly define the responsibilities and rights of various departments. In order to improve the quality of important agricultural cultural heritage protection, local governments need to protect and develop important agricultural cultural heritage in their daily work (you can learn from other types of heritage protection measures). Local governments can strengthen communication with enterprises, communities, and all walks of life, and establish a scientific and reasonable reward system and benefit sharing system to strengthen the supervision and supervision of important agricultural cultural heritage. At this stage, China has formed an important agricultural cultural heritage management mechanism with "government-led, multi-participation, hierarchical management, and classified guidance”.

\subsection{Monitoring and Evaluation of Important Agricultural Cultural Heritage Protection}

The main content of important agricultural cultural heritage management is to monitor and evaluate the protection effect of important agricultural cultural heritage. Therefore, it is necessary to establish a sound evaluation method to comprehensively analyze the significance of important agricultural cultural heritage to the country and the people. At the same time, it is necessary to improve the monitoring mechanism, and regularly or irregularly monitor and evaluate the implementation of important agricultural cultural heritage protection measures and development measures. Apply the effect. At present, China has developed and evaluated agricultural biodiversity cultural diversity, ecosystem service function value evaluation, sustainability evaluation, agricultural cultural heritage tourism resource potential evaluation, and tourism resource development time and space suitability evaluation methods. In addition, China has also thoroughly studied the humanistic characteristics and industrial integration of important agricultural cultural heritage sites.

GIS and geostatistics can be combined to monitor and analyze the spatial differences of soil nutrients in important agricultural cultural heritage sites. In accordance with China's national conditions, China needs to establish a monitoring system that includes agricultural cultural heritage monitoring networks, surveillance and inspection systems, and annual reports on the monitoring of heritage sites. At the same time, it is necessary to pay attention to the professional ability and professional quality of the monitoring personnel to ensure the accuracy of the monitoring results.

\section{Important Agricultural Cultural Heritage Dynamic Protection Mechanism}

\subsection{Protection Object}

The protection of important agricultural cultural heritage is a complex and systematic work that requires the integrity and sustainability of the entire agro-ecosystem, economic system and cultural system. The important cultural heritage protection objects are the various elements involved in traditional agricultural production activities, mainly: traditional agricultural tools, traditional villages, field landscapes, folk culture, traditional knowledge, protection of agricultural technology and other intangible cultural heritage.

\subsection{Protection of the Subject}

The protection of important agricultural cultural heritage cannot be achieved by the efforts of government departments. To ensure the protection effect of agricultural cultural heritage, it is necessary to improve the multi-participation system. In short, it is necessary to establish a 
multi-participation system of government, enterprises, science and technology, community and society. As the responsible person for the protection of important agricultural cultural heritage, the government departments need to coordinate the relationship between the parties to ensure the smooth protection of agricultural cultural heritage; when excavating, protecting and managing the value of important agricultural cultural heritage, important scientific researchers are required to give Science and technology support; the participation of enterprises can promote the smooth protection of important agricultural cultural heritage; the people have the ownership of important agricultural cultural heritage, so they need to directly participate in the protection of important agricultural cultural heritage, which is also the inheritance of agricultural culture and agricultural production activities. The main body of the society participates in the protection of important agricultural cultural heritage, which can effectively enhance the enthusiasm of the people to participate in the protection of important agricultural cultural heritage, and create a good cultural heritage protection atmosphere.

\subsection{Protection Principles and Mechanisms}

Since important agricultural cultural heritage is different from other cultural heritages, it cannot adopt museum-style protection and requires dynamic protection. When protecting important agricultural cultural heritage, it is necessary to pay attention to its changing characteristics. In the dynamic protection of important agricultural cultural heritage, it is necessary to make full use of modern science and technology. While protecting the integrity of the ecological agricultural system, it is also necessary to increase the agricultural income channel and increase the income of farmers in important agricultural cultural heritage sites, thereby promoting important agricultural cultural heritage. Stable and sustainable development. When protecting important agricultural cultural heritage, it is necessary not only to protect the agricultural cultural heritage system, but also to protect the natural environment and human environment around it. Since agricultural cultural heritage sites cannot be relocated, it is necessary to use local conservation methods to protect agricultural cultural heritage, while those responsible for protecting and inheriting agricultural cultural heritage are local farmers.

While clarifying the principles of protection of important agricultural cultural heritage, it is also necessary to establish a law-based incentive mechanism, a scientific support mechanism, a diversified financing mechanism, and a dynamic protection mechanism to enhance the protection of important agricultural cultural heritage and promote important agricultural cultural heritage to continue to develop.

\section{Important Agricultural Cultural Heritage Development Path}

\subsection{Development of Multifunctional Industries}

Agriculture has the characteristics of versatility, which mainly has economic, ecological, social, cultural and other functions. In the process of protecting important agricultural cultural heritage, the characteristics of agricultural versatility can be fully utilized to promote economic stability and sustainable development in agricultural cultural heritage areas. This is also an important reason for all sectors of society to actively participate in the protection of important agricultural cultural heritage. In order to expand agricultural functions, we need to pay attention to the ecological functions and cultural functions of agricultural cultural heritage protection work. Therefore, we need to actively develop agricultural products with cultural connotations and cultural leisure industries with cultural connotations. In order to promote the smooth protection of important agricultural cultural heritage, it is necessary to establish a dynamic protection system including 
agricultural production, agricultural product processing, food and agriculture, biological resources industry, cultural and creative industries. Expand the scope of the agricultural industry and promote the sustainable economic development of important agricultural cultural heritage sites.

\subsection{Development of High Quality Agricultural Products}

Organic foods, green foods, pollution-free products, and specialty products are all high-quality agricultural products. Characteristic products refer to products with geographical compilation and regional public brands in the region. Because the natural ecological environment of important agricultural cultural heritage sites is good, it can produce organic food and green food-grade pollution-free products. By producing high-quality agricultural products, it can improve the farmers' economy in the region while protecting traditional agricultural production techniques and agricultural culture. Income will promote the healthy and sustainable development of the agricultural economy in the region.

Geographical indication products refer to agricultural products that are closely related to the production, quality and reputation of a particular region and their natural environment and human environment. In short, they are legally effective souvenirs. When studying important agricultural cultural heritage, it is necessary to study this in depth. The history, emotion and cultural connotation of regional agricultural culture explore how to integrate agricultural culture with agricultural production, how to build agricultural product brands with regional characteristics, and effectively enhance the economic stability and sustainable development of important agricultural cultural heritage sites. When setting up agricultural brands, we can strengthen cooperation between enterprises, farmers, markets and governments, and improve the economic level of important agricultural cultural heritage sites by deep processing various agricultural products and increasing the value of agricultural products.

\subsection{Development of Agricultural Tourism Industry}

China's important agricultural cultural heritage sites have a large amount of tourism resources. In order to improve the economic level of important agricultural cultural heritage sites, we can actively develop a variety of tourism industries. This will not only stimulate farmers' enthusiasm for participating in the protection of important agricultural cultural heritage, but also help important agriculture. Employment of surplus labor in cultural heritage sites, increase farmers' economic income, and maintain social stability. Different agricultural cultural heritage sites have different folk cultures and characteristics, and their distribution range is wide. The agricultural cultural heritage has high sensitivity and high participation. Therefore, various tourism industries can be developed in agricultural cultural heritage sites. The study found that we can divide the tourism resources of agricultural cultural heritage sites into main elements and auxiliary elements, tangible elements and intangible elements. By studying the versatility of agricultural cultural heritage sites, the tourism development structure of agricultural cultural heritage sites can be formed, which mainly includes the perception of local residents and the distribution of benefits after the development of agricultural cultural heritage sites, the principle of tourism development, and the impact of tourism industry on the ecological environment and so on. At the same time as developing agricultural cultural heritage tourism, it should be noted that it is not the same as rural tourism and agricultural tourism. Agricultural cultural heritage tourism needs to attach importance to the protection and development of agricultural cultural heritage. In recent years, due to the fast pace of people's life and the pressure of work, more and more people choose to travel in their leisure time to relieve their mood. While eco-tourism protects the ecological environment, it can effectively satisfy people's needs for soothing mood. Relying on agricultural cultural heritage sites to develop eco-tourism can 
improve residents' economic income and improve their living standards on the basis of protecting agricultural cultural heritage, which plays an important role in promoting China's economic development and maintaining social stability.

\subsection{Development of Cultural Industries}

In addition to the above points, cultural diversity is also an important feature of agricultural cultural heritage. The inheritance, aesthetics and leisure activities of traditional knowledge will promote the stable development of the cultural industry. We can divide the development path of cultural agriculture into the following categories: First, develop cultural leisure functions. In short, it is necessary to rely on agricultural cultural heritage sites to develop cultural leisure tourism industry, which is of great significance to promote local economic development. However, when it is necessary to pay attention to the development of the tourism industry, it is necessary to coordinate the relationship between folklore and innovation. In the development of tourism based on agricultural cultural heritage, it must abide by the development law of the tourism industry and consumer rights. Second, the development of culture Value added product. In short, it means that agricultural products and agricultural cultural heritage sites are integrated with culture, geography, history and culture to enhance the cultural value of agricultural products, thereby promoting the economic development of agricultural cultural heritage sites and raising the income level of residents in the region. In order to develop cultural industries, it is necessary to strengthen the inheritance and protection of the culture of agricultural cultural heritage sites. Therefore, it is necessary to strengthen cultural protection and reward systems, and promulgate government support policies and other measures to strengthen cultural protection.

\section{Conclusion}

The protection of agricultural cultural heritage is to ensure the stability and development of agricultural regions with profound cultural heritage and economic backwardness while ensuring the diversity of agricultural biodiversity and agricultural culture. China has achieved a certain degree in the study of agricultural cultural heritage, which is of great significance to ensure the mining and protection of agricultural cultural heritage in China. China has established a specialized research team, which is important promote for the protection and utilization of agricultural cultural heritage in China. In the future, China needs to not only strengthen the protection of agricultural cultural heritage, but also promote the stable and sustainable development of agriculture.

\section{References}

[1] Quanyong Li. Let Agricultural Cultural Heritage Become a New Kinetic Energy for Rural Development [J]. Agricultural Knowledge, 2017(26): 23-24.

[2] Qingwen Min, Yongxun Zhang. Comparative Study on Agricultural Cultural Heritage and Agricultural Cultural Landscape Heritage[J]. Journal of China Agricultural University, Social Sciences, 2016, 33(2): 119-126.

[3] Yehong Sun, Qingwen Min. Several Misunderstandings in Tourism Development of Agricultural Cultural Heritage Sites [J]. Wisdom China, 2017(8): 92-93.

[4] International Exchange Service Center of the Ministry of Agriculture. Positive achievements in Global Important Agricultural Cultural Heritage Work [J]. World Agriculture, 2017(7): 246-246.

[5] Yizhi Wang, Wenqian Chen. On the Relationship Between the Protection of Agricultural Cultural Heritage and Tourism Development in Zhaina[J]. World Agriculture, 2017(10): 175-181.

[6] Zongwei Han. Analysis of Spatial Distribution Characteristics and Influencing Factors of Chinese Agricultural Cultural Heritage [J]. Chinese Journal of Agricultural Resources and Regional Planning, 2017, 38(2): 97-104. 